\title{
Von Interaktion zu Transaktion - Konsequenzen eines pragmatischen Mensch-Umwelt-Verständnisses für eine Geographie der Mitwelt
}

\author{
C. Steiner \\ Institut für Geographie, Universität Osnabrück/Institut für Humangeographie, Goethe Universität Frankfurt, \\ Germany
}

Correspondence to: C. Steiner (christian.steiner@uni-osnabrueck.de)

Received: 8 October 2013 - Revised: 29 January 2014 - Accepted: 13 February 2014 - Published: 8 October 2014

\begin{abstract}
Kurzfassung. Questions about how human-environment-relations can be conceptualized in a non-dualistic way have been intensively discussed throughout the last decades. The majority of the established realist and constructivist perspectives aim at explaining a given situation by analytically dissecting it. Unfortunately, such an interactionist perspective systematically reproduces the dualistic division between humans, environment and nature.

In contrast, this paper offers a transactive perspective origin in classical pragmatism and discusses its metatheoretical consequences for human-environment-research. A transactionist perspective interprets the world as a flow of unique and entangled events. Instead of ontologically separating humans and environment, it advocates to look at their relations as being part of a "connatural world". Such a point of view raises new ethical and political questions for geographical human-environment research, argues for a renaissance of ideographic methodologies and hints to a fruitful unity of geographical inquiry.
\end{abstract}

\section{Einleitung}

Die Frage wie Mensch-Umwelt-Beziehungen produktiv konzeptionell gefasst werden können beschäftigt die MenschUmwelt-Forschung innerhalb (vgl. für einen Überblick bspw. Meusburger and Schwan, 2003; Müller-Mahn and Wardenga, 2005; Wardenga and Weichhart, 2006; Weichhart, 2005) und außerhalb (bspw. Hirsch, 1995; Becker and Jahn, 2006) der Geographie nun schon seit vielen Jahren.

Das Problem der integrativen Konzeptionalisierung von Mensch-Umwelt-Beziehungen beschränkt sich dabei keineswegs auf eine angemessene fachtheoretische oder methodische Annäherung (Müller-Mahn and Wardenga, 2005:5). Vielmehr gründet es auch in den dualistischen Weltbildern von Realismus und Konstruktivismus, die eine Erkenntnis über die Welt entweder aus rationalistischer oder aus empiristischer Perspektive denken und das Problem insofern spiegelbildlich angehen. In der Mensch-Umwelt-Forschung manifestiert sich diese Frontstellung von Geist und Materie in einer Denkfigur, die Mensch und Umwelt getrennt als eigenständige Entitäten denkt. Aus dieser Sicht bestimmt sich der
Sinn jeder Mensch-Umwelt-Forschung logischerweise darin, danach zu fragen, wie Mensch und Umwelt interagieren. Durch diese interaktionistische Perspektive wird die Trennung von Mensch und Umwelt jedoch systematisch reproduziert. Realismus und Konstruktivismus sind daher ungeeignet, um zu wirklich integrativen Ansätzen zu finden (Steiner, 2009).

Zunehmend wird die Sinnhaftigkeit einer dichotomen Grenzziehung zwischen Kultur und Natur sowie Mensch und Umwelt innerhalb und außerhalb der Geographie jedoch in Frage gestellt (bspw. Descola, 2011; Falter and Hasse, 2002; Jöns, 2003; Latour, 2010; Schlottmann et al., 2010; Whatmore, 2002; Zierhofer, 1999). Auch in der Geographie nehmen daher die Bemühungen um die Entwicklung ,postdualistischer Ansätze“ zu (Graefe et al., 2010).

Was in der bisherigen Diskussion jedoch zu kurz gekommen zu sein scheint, ist auch nach neuen metatheoretischen Wegen zu suchen, mit denen es möglich ist, das Verhältnis von Mensch und Umwelt auf eine nichtdualistische Weise zu bestimmen. Die bisher hierzu diskutierten Ansätze im Bereich der Sprachpragmatik, Phänomenologie, 
Actor-Network-Theory (ANT) und Non-RepresentationalTheory (NRT) bleiben in ihrer erkenntnistheoretischen Fundierung nämlich in Teilen vage oder problematisch, wie im nächsten Abschnitt noch diskutiert werden wird. Beides zieht jedoch erhebliche wissenschaftstheoretische Probleme für eine in sich konsistente Bestimmung von Forschungsperspektiven, Forschungsgegenständen und Methodologien nach sich.

Der vorliegende Artikel möchte daher zu einer Stärkung der Kohärenz nichtdualistischer Theorien in der geographischen Mensch-Umwelt-Forschung beitragen, indem er der Frage nachgeht, wie Mensch-Umwelt-Beziehungen jenseits dualistischer und interaktionistischer Perspektiven auf metatheoretischer Ebene gedacht werden können. Dazu skizziert er zunächst kurz, inwiefern den bisher diskutierten nichtdualistischen Ansätzen eine - wie oben behauptet - metatheoretische Unbestimmtheit inne wohnt. Danach stellt er dem die Welt fragmentierenden dualistischen Konzept der Interaktion das aus dem klassischen Pragmatismus stammende holistische Konzept der Transaktion gegenüber und eröffnet eine Diskussion über dessen meta-theoretische Konsequenzen für veränderte Forschungsperspektiven, Fragestellungen und Methodologien in der geographischen Mensch-UmweltForschung.

\section{Die metatheoretische Unbestimmtheit nichtdualistischer Theorien}

Die in der humangeographischen Diskussion befindlichen nichtdualistischen Ansätze der Sprachpragmatik, Phänomenologie, ANT und NRT haben auf der fachtheoretischen Ebene zweifellos vielversprechende Ansatzpunkte für die Ausgestaltung einer postdualistischen Geographie aufgezeigt. Für eine metatheoretische Bestimmung des Verhältnisses von Mensch und Umwelt sind sie aber nur bedingt geeignet.

Metatheoretisch am problematischsten erscheint dabei der Versuch sprachpragmatische Ansätze im Anschluss an Austin, Searle und Rorty für die Entwicklung einer nichtdualistischen Mensch-Umwelt-Geographie nutzbar zu machen (bspw. Schlottmann, 2007; Zierhofer, 1997). Zierhofer (1997:89) räumt hierzu selber ein, dass eine Konzentration auf sprachpragmatische Ansätze das Problem mit sich bringe, sich damit des erfahrungsbasierten und insofern empirischen Zugangs zur Welt außerhalb von Sprachspielen zu berauben. Während nämlich Austin (1962) sich vor allem für die performative Wirkung von Sprache interessiert und die empirische Erfahrung von Materialität deshalb nicht in seinem Fokus steht, lehnt Rorty (1994:25) sogar jede Bezugnahme auf Erfahrung ab, da diese für ihn auf eine Außenwelt verweist, über die wir seiner Meinung nach keine Erkenntnis erlangen können. Searles kritisch-realistische Grundhaltung bietet hier zwar, wie Schlottmann (2007:12) anmerkt, mehr Möglichkeiten, Materialität praktisch zu fassen. Unbestimmt bleibt dabei aber, wie sich der implizit im Realismus ange- legte Dualismus zwischen Realität und Repräsentation nichtdualistisch auflösen lässt.

Die phänomenologische Mensch-Umwelt-Forschung (bspw. Falter and Hasse, 2002) bricht mit den gängigen Dualismen zwischen Mensch und Umwelt vor allem auf der Ebene der Theoriebildung und empirischen Arbeit, in dem sie die Bereiche der Gefühle, Sinnlichkeit und Ästhetik über ihre Schlüsselfunktion für das Erleben der Welt in den Fokus der Aufmerksamkeit rückt. Das Theoriegebäude der Phänomenologie bricht insofern zwar mit zahlreichen Dualismen, bleibt aber in ihrer erkenntnistheoretischen Basis entweder, wie Husserl, einer idealistischen Perspektive oder, wie Hartmann, einer realistischen Metaphysik verhaftet (Hügli and Lübcke, 2005:490). Erst mit Schütz' Theorie der Lebenswelt (Schütz and Luckmann, 2003) löst sich die Phänomenologie von idealistischen und realistischen Fundierungen, indem sie pragmatische Elemente in ihre Theorieentwicklung mit aufnimmt. Wie genau MenschUmwelt-Verhältnisse erkenntnistheoretisch zu denken sind, bleibt dabei allerdings offen.

Auch ANT und NRT eignen sich nicht, um das erkenntnistheoretische Problem in der Konzeptionalisierung von Mensch-Umwelt-Beziehungen zu lösen, da sie sich explizit nicht als epistemologische, sondern als sozialwissenschaftliche und politische Diskussionsbeiträge verstehen, mit denen sie das Verhältnis des Sozialen zu seiner Umwelt neu bestimmen wollen (Latour, 2010:31; Thrift, 2008:2). Auch wenn sie diesbezüglich recht vage bleiben, verweisen Latour wie auch Thrift jedoch an verschiedenen Stellen in ihren Arbeiten auf Einflüsse des klassischen Pragmatismus auf ihre Theorieentwicklung. Latour beruft sich vor allem auf William James als Inspiration für die Entwicklung seiner DingGeschichte (1990:79f) und formuliert hiervon ausgehend in Anlehnung an Alfred Whitehead seine wissenschaftstheoretische Position für die Science Studies (1996:89; 2002:137ff; 2012:412). Thrift lehnt sich in ähnlicher Weise an James und Whitehead an (2008:6), greift aber auch auf John Deweys Idee des ,embodiment“" kultureller Praktiken und Ästhetiken zurück und baut damit eine Brücke zu den gegenwärtig in der Humangeographie intensiv diskutierten Performativitätskonzepten (2008:148f).

Die genannten Ansätze in der nichtdualistischen Theoriedebatte der Mensch-Umwelt-Forschung lösen sich insofern entweder in ihrer erkenntnistheoretischen Basis nicht vollständig von dualistischen Denkfiguren und Ontologien oder sie bleiben diesbezüglich vage. In erkenntnistheoretischer Hinsicht wird zwar immer wieder latent auf den klassischen Pragmatismus Bezug genommen, seine metatheoretische Konzeption von Mensch-Umwelt-Beziehungen und ihre wissenschaftstheoretischen Konsequenzen haben bislang jedoch kaum direkten Eingang in die Debatte gefunden. 


\section{Pragmatismus und Mensch-Umwelt-Forschung}

Die weitgehende Abwesenheit des Pragmatismus in der geographischen Mensch-Umwelt-Forschung (Wood and Smith, 2008:1527) ist umso erstaunlicher, als sich historisch durchaus enge Verbindungen zwischen Pragmatismus, Geologie und Geomorphologie ausmachen lassen (Baker, 1996).

Auf die Rezeption des pragmatischen Transaktionskonzeptes in der Umweltpsychologie wird Weichhart im deutschsprachigen Raum zu Beginn der 1990er Jahre aufmerksam und diskutiert deren Potenziale für die Humanökologie (1991, 1993). Interessanterweise setzt er sich mit dem Originalentwurf des Transaktionskonzeptes durch John Dewey jedoch nicht auseinander. Weichharts Veröffentlichungen bleibt - eventuell auch wegen der starken Bezüge zur Umweltpsychologie - eine breitere Resonanz innerhalb der Geographie jedoch versagt.

Wescoat (1992) arbeitet fast zeitgleich den Einfluss von Dewey auf die Arbeiten von White über natürliche Ressourcen und Naturgefahren heraus. Proctor (1998b) versucht pragmatische und sozial-konstruktivistische Ansätze zusammenzuführen und so ein neues Verständnis von Natur in der Geographie anzuregen und diskutiert hiervon ausgehend (1998a) die Bedeutung umweltethischer Fragen für die Geographie. In gleicher Art betont auch Hobson (2006) die Fruchtbarkeit des Pragmatismus für die Untersuchung umweltverantwortlichen Handelns im Rahmen einer ethisch orientierten Nachhaltigkeitsforschung.

Etwas grundlegender diskutieren Hepple (2008) und Barnes (2008) neben anderen Themen auch die erkenntnistheoretische Position des Pragmatismus in Bezug auf ein nichtdualistisches Mensch-Umwelt-Verständnis. Die Auflösung der Geist-Körper-Dichotomie thematisiert Bridge (2008) vor dem Hintergrund der Konstitution städtischer Räume. Elemente von Deweys Metaphysik klopft Cutchin (2008) u.a. hinsichtlich ihrer Implikationen für das Verständnis von Mensch-Umwelt-Beziehungen ab und diskutiert deren Folgen für eine Rekonzeptionalisierung von „Place“ in der Geographie. Auf grundsätzlicherer Ebene erörtern lediglich Proctor (1998b) und Steiner (2009) die erkenntnistheoretisch andersartige Konzeption von Mensch und Umwelt sowie Materie und Geist im Pragmatismus und grenzen sie ab gegenüber Konstruktivismus und Kritischem Realismus.

Obwohl insofern vereinzelt auf das Potenzial des Pragmatismus für eine geographische Mensch-Umwelt-Forschung hingewiesen wird, steht eine theoretisch gesättigte ErschlieBung des Transaktionskonzeptes für die Geographie noch immer aus.

\section{Von Interaktion zu Transaktion}

Das Transaktionskonzept ist innerhalb der Philosophie John Deweys - als einem der Hauptvertreter des klassischen Pragmatismus - das entscheidende theoretische Scharnier, um verstehen zu können, wie der Pragmatismus das Verhältnis von Menschen zu ihrer Umwelt denkt. Dies ist deshalb besonders wichtig, weil die Auseinandersetzung von Menschen mit ihrer Umwelt einen der Hauptfoki des klassischen Pragmatismus bildet.

Die Idee der Transaktion ist jedoch recht sperrig. Ihre Entwicklung und Ausformulierung zieht sich zudem in Deweys Werk über einen Zeitraum von fast 25 Jahren hin. Erste Anlagen zu seiner Transaktionstheorie lassen sich bereits seinem Buch „Experience and Nature“ (1925) entnehmen. Seine Gedanken zu dem Verhältnis von Körper und Geist sowie Mensch und Umwelt entwickeln sich dann u.a. in dem Essay „Body and Mind“ (1927) und einem seiner weiteren Hauptwerke „Logic: The Theory of Inquiry“ (1938) weiter, bis er, schon fast am Ende seines Lebens, den Versuch unternimmt, sein Konzept der Transaktion zusammen mit Arthur F. Bentley in „Knowing and the Known“ (1949) genauer auszuformulieren.

Um sein Konzept thematisch aufzuschließen bietet es sich an, mit Deweys und Bentleys (1949:LW.16.4) Unterscheidung von Selbst-Aktion (self-action), Interaktion (interaction) und Transaktion (transaction) zu beginnen. Alle drei Modi stellen für Dewey und Bentley (ebd.:LW.16.100) Ebenen der Organisation und Präsentation menschlichen Forschungsverhaltens in und mit Bezug auf die Welt dar, in der sich das menschliche Verständnis der Welt spiegelt.

In der Perspektive der Selbst-Aktion werden Dinge betrachtet, als wirkten sie durch eigene Kräfte (ebd.). Voraussetzung hierfür ist die Idee, dass es unabhängig existierende Entitäten wie Akteure, das Bewusstsein, das Ich und ihnen innewohnende Kräfte und Fähigkeiten gibt, die Ereignisse auslösen können (ebd.:LW.16.71).

Demgegenüber versteht Interaktion das Verhältnis der Dinge als aufeinander abgestimmte Beziehung, die durch kausale Verbindungen geprägt ist (ebd.:LW.16.100). Der wesentliche Unterschied zwischen den Perspektiven von SelbstAktion und Interaktion besteht darin, dass in letzterem die Dinge so organisiert werden, dass sie sich als auf der Basis des jeweils anderen operierend präsentieren. Wir finden hier keine eigenständigen Entitäten, sondern scheinbar in sich geschlossene und unabhängige, effizient arbeitende Systeme, wie die newtonsche Mechanik. Typisch für diese bspw. in den Naturwissenschaften und der Medizin verbreitete Perspektive ist es, Elemente und ihre Beziehungssysteme aus einem größeren Zusammenhang zu isolieren, um einzelne Funktionszusammenhänge genauer untersuchen zu können. Eine interaktionistische Denkweise geht daher mechanistisch, reduktionistisch und kausalistisch an die Welt heran und will durch die Zerlegung der komplexen Wirklichkeit in einzelne Teile Erkenntnisse gewinnen. Stillschweigend geht man davon aus, dass ein Verständnis des Gesamten durch die Untersuchung von Teilsystemen und deren Wechselwirkung möglich ist. Das Gesamte wird daher als die Summe seiner Teile gedacht.

Nicht nur mit Blick auf soziale sondern auch bzgl. medizinischer oder naturwissenschaftlicher Fragestellungen 
drängt sich jedoch der Einwand auf, dass Forschungsergebnisse nur im Zusammenhang des jeweiligen Gesamtgefüges sinnvoll interpretiert werden können (ebd.:LW.16.103). Hier schließt die Perspektive der Transaktion an. Transaktion meint die funktionale Beobachtung eines holistischen und sich dynamisch wandelnden Ganzen (full subjectmatter) (ebd.:LW.16.113) mit für Veränderungen der Beschreibungen offenen Begriffen und Benennungen. Sie geht davon aus, dass komplexe Gegenstände Eigenschaften besitzen, die sich nicht allein aus der Summe ihrer Teilelemente ableiten lassen. Einzelaspekte eines Gesamtgefüges lassen sich lediglich in Relation zu dessen anderen Teilen bestimmen. Es ist nicht möglich, Teile des Ganzen als eigenständige Elemente abzugrenzen und für sich zu fixieren. Der Blick auf Forschungsgegenstände verändert sich damit dahingehend, dass es nicht mehr möglich ist, sie von ihrem Umfeld und ihrer Umwelt loszulösen (ebd.:LW.16.71). Transaktion repräsentiert deshalb eine Perspektive auf Forschungsprozesse, in der Beobachtungen beschrieben werden, ohne einzelne Phasen oder Aspekte des untersuchten Ereignisses ontologisch zu isolieren und unabhängigen Akteuren, Elementen oder interagierenden Systemelementen zuzuschreiben (ebd.:LW.16.112).

Der Unterschied zwischen einer interaktionistischen und transaktionistischen Perspektive ist insofern grundsätzlicher als es auf den ersten Blick scheinen mag. Während Interaktion eine Perspektive auf die praktisch-forschende Auseinandersetzung mit der Umwelt beschreibt, in der Ereignisse unter der Prämisse betrachtet werden, dass sie adäquat und korrekt beschrieben worden wären bevor man die in ihnen wirkenden Verbindungen erforscht, versteht Transaktion jede Art von Beschreibung nur als ersten, vorläufigen und tastenden Versuch, eine Situation sprachlich zu erfassen; eine Situation, deren Beschreibung sich im Verlauf der weiteren Forschungsprozesse zu jeder Zeit immer wieder verändern kann (ebd.:LW.16.113). Wichtig ist es deshalb, möglichst Beschreibungs- und Benennungssysteme zu verwenden, die nicht begrifflich auf isolierte Elemente, letzte oder unabhängig existierende Dinge, Entitäten, Wesen oder Realitäten verweisen und die die Beziehungen zwischen den Dingen von den Dingen nicht abtrennen (ebd.:LW.16.101f).

Mit ihren Begriffen sind auch die vordefinierten Forschungsobjekte grundsätzlich offen für Veränderungen, denn sie müssen sich im Verlauf von Forschungsprozessen wandeln können, wenn sich das Verständnis des Ganzen weiterentwickelt (ebd.:LW.16.71, LW.16.113f). Forschungsobjekte existieren aus dieser Perspektive daher nicht bereits vor der Auseinandersetzung mit der Welt, sondern werden begrifflich operational geschaffen. Sie sind das Produkt situierter menschlicher Aktivität. Die Unterscheidung zwischen vermeintlich stabilen Zuständen, die wir Dinge nennen, und ihren Veränderungsprozessen wird somit kontingent. Eine transaktionale Perspektive zwingt einen deshalb den vorläufigen, kontingenten und konstruierten Charakter jeder Beschreibung zur Kenntnis zu nehmen (Garrison, 2001:287).

\subsection{Von Dingen zu Ereignissen}

Als Konsequenz des Transaktionskonzeptes muss sich auch das Verständnis von Dingen radikal verändern. Dinge können nichts Statisches mehr sein, wie aus Perspektive der Interaktion. Unterscheidungen von Dingen (thing) und Aktivitäten (action) haben allenfalls provisorischen Charakter (Dewey and Bentley, 1949:LW.16.113). Was wir Ding nennen ist für Dewey nichts anderes als eine Art zeitlich und räumlich ausgedehntes Ereignis (event), ein analytisch isolierbares Element in einem transaktiven Prozess permanenten Wandels. Gegenwärtige Ereignisse verändern dabei immer reflexiv die Bedingungen zukünftiger Prozesse. Alles, um mit Parmenides zu sprechen, ist im Fluss. Die Welt ist geprägt von emergenten Prozessen, die eine historische Abfolge situativ einzigartiger Ereignisse hervorbringen. Die Natur besteht deshalb aus einer Abfolge einmaliger geschichtlicher Ereignisse (Dewey, 1925:LW.1.5f). Auf Grund der räumlichen, zeitlichen und situativen Einzigartigkeit dieser Prozesse und ihrer reflexiven Wirkung entziehen sich Veränderungen des Ganzen dann aber logisch einer exakten Prognostizierbarkeit.

Der historische Weg zu einer dynamisch transaktiven Perspektive war lang. Schon in der griechischen Antike entwickelte sich eine dualistische Perspektive auf Welt, die von der Idee feststehender, unendlicher Ideen bzw. Substanzen ausging. Besonders der starke Einfluss von Aristoteles in den empirischen Wissenschaften führte dazu, dass die Dinge, und gerade die Dinge der Umwelt, in der wir leben, als Substanzen gedacht wurden, die ein eigenes Wesen besitzen und grundsätzlich stabil sind, wenn nicht äußere Kräfte auf sie wirken. Hieraus entstanden die interaktionistischen Theorien.

Es verwundert daher nicht, dass Deweys Dynamisierung und Prozessualisierung der (Natur-) Philosophie auf Widerstände traf. Die Idee, dass die Dinge der Welt als zeitlich und räumlich ausgedehnte Ereignisse zu begreifen seien, muss zu ihrem Entstehungszeitpunkt noch viel befremdlicher geklungen haben als heute. Dass Dewey seine Idee der Existenz als Ereignis gerade an einem Beispiel aus den Geowissenschaften veranschaulicht, mag insofern kein Zufall sein:

Even the solid earth mountains, the emblems of constancy, appear and disappear like the clouds. A thing may endure yet not be everlasting; it will crumble before the gnawing tooth of time, as it exceeds a certain measure. Every existence is an event (Dewey, 1925:LW.1.63).

Es verbietet sich daher Ereignisse mit Substanzen zu verwechseln, da erstere nicht (in sich) abgeschlossen, nicht unvergänglich, sondern endlich, unvollständig und unabgeschlossen sind (ebd.:LW.1.126f). Ereignisse haben deshalb auch keine präfixierte Essenz oder Bedeutung. Bedeutungen bilden sich vielmehr erst als Konsequenz transaktionaler Forschungsprozesse heraus, in denen Ereignisse als in Raum und Zeit verortet wahrgenommen und im 
Forschungsprozess als Objekte aktiv operational fixiert werden (Dewey, 1938:116ff). Auch wenn Objekte insofern als im Forschungsprozess geronnene Ereignisse aufgefasst werden können, verlieren sie nie gänzlich ihre Ereignisqualität (Garrison, 2001:288). Objekte sind dann eher als Fakten, als etwas Gemachtes, zu verstehen, das ohne den Verweis auf andere Elemente nicht sinnvoll gedacht werden kann (Dewey and Bentley, 1949:LW.16.113). Die Welt und ihre Dinge sind daher immanent dynamisch und relational angelegt.

\subsection{Organismus und Umwelt}

Die transaktionistische Konzeption von Dingen hat Folgen für die Art und Weise, wie das Verhältnis von Organismen und Umwelt gedacht werden muss. Die Unterscheidung von Organismus und Umwelt ist vor dem Hintergrund einer transaktionistischen Denkfigur nämlich nicht mehr trennscharf aufrecht zu erhalten. Da ein Organismus sich in seinen Aktivitäten immer auf seine Umwelt als Abfolge räumlich und zeitlich ausgedehnter Ereignisse bezieht, sind seine vergangene und gegenwärtige Umwelt impliziter Teil seines aktuellen Verhaltens und tragen dazu bei, es zu dem zu machen, was es ist. Es ist weder allein durch interne noch durch externe Prozesse und Strukturen erklärbar, sondern resultiert vielmehr aus einer Integration von Organismus und Umwelt (Dewey, 1996:LW.1.213), die sich im Umstand des Lebens selbst begründet:

Life is (...) a process of activity that involves an environment. (...) An organism does not live in an environment; it lives by means of an environment. Breathing, the ingestion of food, the ejection of waste products, are cases of direct integration; the circulation of the blood and the energizing of the nervous system are relatively indirect. But every organic function is an interaction of intraorganic and extra-organic energies (...). For life involves expenditure of energy and the energy expended can be replenished only as the activities performed succeed in making return drafts upon the environment-the only source of restoration of energy. (...) The processes of living are enacted by the environment as truly as by the organism; for they are an integration (ebd.:LW.12.32).

Nimmt man die Verbindung von Organismus und Umwelt ernst, wird die Grenze zwischen ihnen unscharf. Was gerade noch Umwelt war wird im nächsten Augenblick eingeatmet oder absorbiert, in den Organismus eingebaut oder von ihm abgesondert. Was Organismus und was Umwelt ist, variiert deshalb im Laufe der Zeit (Garrison, 2001:291).

Der Unterscheidung von Organismus und Umwelt liegt insofern ebenfalls die analytische Isolation eines zeitlich und räumlich ausgedehnten Ereignisses zu Grunde, das in einen größeren Ereigniskomplex integriert und ohne diesen nicht vollständig $\mathrm{zu}$ verstehen ist. Wir können ein einziges Or- gan in seiner Funktion genauso wenig verstehen, wie die Existenz eines ganzen Organismus, wenn wir seine Einbettung in einen größeren Zusammenhang ausblenden. Es ist das Leben selbst, das funktional koordinierte Transaktionen bedingt. Ein Organismus ist also letztlich, wie Dewey und Bentley (1949:LW.16.103) es ausdrücken, immer nur als ein transaktiver organism-in-environment-as-a-whole versteh- und denkbar.

Die Unterscheidung zwischen Organismus und Umwelt wird damit zwar arbiträr, sie ist jedoch nicht weniger legitim. Ihre Legitimität kann sie aber nicht mehr aus einer ontologischen, sondern nur noch aus einer methodologischen Begründung heraus beanspruchen.

Das Verständnis von Umwelt wandelt sich in dieser Perspektive hin zu dem eines Mediums oder Milieus, in dem und durch das Organismen leben. Umwelt ist daher nichts einem Organismus - und damit auch dem Menschen als organische Spezialform - Externes, sondern integraler Teil seiner Existenz (Dewey and Bentley, 1949:LW.16.244).

\subsection{Trans-Akteure, Erfahrung und Kultur}

Wenn Organismen und Umwelt nur analytisch unterschieden werden können, muss sich auch das Verständnis von Akteuren hin zu Trans-Akteuren verändern (ebd.:LW.16.260). Auch Menschen sind in diesem Sinn als transaktionale, individuelle Ereignisse zu verstehen. Akteure als Trans-Akteure müssen demnach als eine Art temporal isolierte Phase einer Aktivität begriffen werden, die ihre eigene Aktivität danach ausrichten, das dynamische und sich im kontinuierlichen Wandel befindliche Gesamte funktional zu beeinflussen und in ihrem Sinne zu verändern. Das Subjekt steht als Trans-Akteur demnach seiner Umwelt nicht mehr distanziert und unbeteiligt im Erkenntnisprozess gegenüber, sondern ist transaktiv mit ihr verwoben. Umwelt ist dann das, was die Funktionalität des Gesamten, von dem Trans-Akteure ein Teil sind, sowohl stört, behindert wie auch stützt (Dewey, 1996:LW.1.216).

In einem solchen dynamischen Verständnis von TransAkteur und Umwelt wäre es ein Fehler, Umwelten auf ein Bündel physisch-materieller Bedingungen $\mathrm{zu}$ reduzieren. Physisch-materielles ist vielmehr immer als verwoben mit Kultur zu denken. Alles andere würde auf einen kruden Naturdeterminismus und stark vereinfachenden Naturalismus herauslaufen (Hampe, 2006:122), von dem die MenschUmwelt-Forschung in der Geographie in weiten Teilen zu Recht schon lange Abstand genommen hat. Aus Sicht des Pragmatismus muss man solche Naturalismen mit dem Verweis auf die Ideen der Abduktion und Deduktion (Steiner, 2009) auch klar zurückweisen, denn die Dinge der Welt haben für uns immer eine Bedeutung, die im Rahmen von Erfahrungsprozessen entsteht. Erfahrung gleicht dabei einem Organisationssystem des Wissens über die Welt (Dewey and Bentley, 1949:LW.16.52) und insofern einem Werkzeug, mit 
dessen Hilfe Menschen in ihre Umwelt vordringen (Dewey, 1996:LW.1.5).

Nimmt man die transaktive Beziehung zwischen Organismus und Umwelt ernst, ist es deshalb unsinnig, die Erfahrung von Umwelt von dieser Welt ontologisch zu trennen (Dewey, 1925:LW.1.19). Eine naturalistische Trennung der erfahrenen Umwelt von dem Akt des Erfahrens würde letztlich wieder zu einem Denken in Wesenheiten und Substanzen zurückführen. Da menschliche Erfahrung jedoch sowohl von unserer physischen Existenz wie auch kulturell geprägt ist, muss auch die Umwelt als unser Medium immer sowohl physisch-materiell wie auch zugleich kulturell gedacht werden (Dewey and Bentley, 1949:LW.16.244).

Aus einer transaktiven Perspektive ergibt es deshalb auch keinen Sinn, dass Subjekt quasi zu entwirklichen und es (wie in idealistischen Subjektivitätstheorien und einigen konstruktivistischen Positionen) mitsamt den kulturell geprägten Bedeutungen, die die Dinge für das Subjekt haben, der physisch-materiellen Welt antipodisch entgegenzusetzen (Hampe, 2006:122). Kultur und Umwelt sind in transaktiver Perspektive vielmehr co-konstitutitiv für einander.

\subsection{Body-Minds als Auflösung des Geist-Materie-Dualismus}

Dass Umwelt und Natur kulturell geprägt sind, ist die Seite transaktionalen Denkens, die interaktionistisch denkende Realisten am ehesten irritieren mag. Dass die transaktive Beziehung zwischen Umwelt und Organismus auch Konsequenzen für die Konzeption des Bewusstseins und des Verstandes impliziert, mag wiederum bei Konstruktivisten auf Befremden stoßen. Denn Geist und Körper sind für den Pragmatismus gemeinsam zu denken:

Every „mind“ that we are empirically acquainted with is found in connection with some organized body (Dewey, 1996:LW.1.212).

Den Gedanken der transaktiven Einheit von Körper und Geist entwickelt Dewey bereits in seinem Essay „Body and Mind“ und veranschaulicht deren Verbindung am Beispiel des Essens:

The being (...) eats and digests in one way to one effect when glad, and to another when he is sad. Eating is also a social act and the emotional temper of the festal board enters into the alleged merely physical function of digestion. Eating of bread and drinking of wine have indeed become so integrated with the mental attitudes of multitudes of persons that they have assumed a sacramental spiritual aspect. (...) The case of taking and assimilating food is (...) an act in which means employed are physical while the quality of the act determined by its consequences is also mental. (...) Instead of taking the act in its entirety we cite the multitude of relevant facts only as evidence of influence of mind on body and of body on mind, thus starting from and perpetuating the idea of their independence and separation even when dealing with their connection (Dewey, 1996:LW.3.28f).

Das Beispiel veranschaulicht die Einheit von Körper und Geist, deren Trennung künstlich erscheint, denkt man intensiver über sie nach. Dies wird umso deutlicher, wenn man sich vor Augen führt, dass die holistische Einheit menschlichen Lebens geradezu Voraussetzung von Handlungsfähigkeit ist (Dewey, 1996:LW.3.28). Während der Körper für die Handlungsmittel und Möglichkeiten des Handlungsvollzugs steht, steht der Geist für dessen Plan, Früchte und Konsequenzen (ebd.:LW.3.31). Es braucht die Einheit von Körper und Geist, um reden, zuhören, denken, riechen, fühlen oder sehen zu können. Damit brechen die traditionellen Grenzen zwischen Geist und Körper sowie Wissen und Praxis jedoch zusammen (Dewey, 1996:LW.3.27). Begrifflich versucht Dewey (ebd.:LW.3.27) die neu gedachte holistische Einheit von Körper und Geist mit dem Ausdruck des Body-Mind oder Mind-Body zu fassen.

Rein Mentales, Sinn, kann es in dieser Perspektive ohne Materie nicht geben. Das Gedachte, als auf etwas Verweisendes und in diesem Sinne als Zeichen, könnte, ohne Teil eines transaktiven Prozesses zu sein, weder existieren noch mitgeteilt werden (Dewey and Bentley, 1949:LW.16.138). Es ist deshalb weder losgelöst von dem Denkenden noch von seiner Entstehungssituation sinnvoll zu betrachten. Bedeutungen, als der Inhalt des Gedachten, und der Verstand sind co-konstitutiv. Sie entstehen für Dewey gleichzeitig in einem Prozess transaktionaler, funktionaler Koordination in der forschenden Auseinandersetzung des Organismus mit seiner Umwelt (ebd.:LW.1.230). Der Verstand werde erst als etwas Entkörperlichtes wahrgenommen, wenn Menschen sich mit anderen Menschen kommunikativ austauschen und dabei Zeichen und ihre Bedeutungen auf einmal als vermeintlich unabhängig von ihrem Entstehungskontext (und ihren Konsequenzen) wahrnehmen (ebd.:LW.1.198). Dewey und Bentley (1949:LW.16.266) distanzieren sich daher von ,disembodied meanings and meaningless bodies".

Konsequenterweise weisen daher Dewey und Bentley (1949:LW.16.112) auch die konstruktivistische Idee, dass Sprache eine eigene Wirklichkeit ohne Bezug zur materiellen Umwelt konstituiere, entschieden zurück:

We reject the „no man's land“ of words imagined to lie between the organism and its environmental objects (...) and require, instead, definite locations for all naming behaviors as organic-environmental transactions under observation.

Sprache ist also für Dewey und Bentley nichts der Welt externes, wie die Analytische Philosophie oder der Logische Positivismus russellscher Prägung postulieren. Worte verstehen sie stattdessen als operationale Mittel, um in eine bestimmte Art der Transaktion mit der Welt einzutreten. 
Deweys Idee der Body-Minds verwurzelt insofern mentale und sprachliche Akte wieder in der Welt. Diskurse und Texte, wie viele Poststrukturalisten, so zu behandeln als führten sie ein Eigenleben ohne Sprecher und Rezipienten ergibt dann keinen Sinn (Garrison, 2001:295). Im Konzept der Body-Minds sind Diskurse vielmehr elementarer Bestandteil der praktischen, transaktiven, forschenden Auseinandersetzung von Menschen mit ihren (sozialen) Umwelten (Garrison, 2001:292).

\section{Konsequenzen einer transaktionistischen Perspektive für die Konzeptionalisierung von Mensch-Umwelt-Beziehungen}

Eine transaktionistische Perspektive bricht, wie gezeigt wurde, auf breiter Front mit gewohnten interaktionistischen Perspektiven. Als Metatheorie ist sie aber natürlich nicht einfach eins-zu-eins in die Geographie importierbar. Barnes (2008:1551) hat in diesem Sinne schon darauf hingewiesen, dass es inadäquat wäre zu erwarten, dass man den Pragmatismus einfach wie eine neue Art Erklärungsmaschine für geographisch relevante Gegenstände benutzen könnte und heraus käme dann die Pragmatische Geographie. Wie Menand (2001:xi) ausführt, ist der Pragmatismus auch keine ,große Idee", die alles erklären könnte. Sie ist eher eine Art Werkzeug des Nachdenkens über das Denken, das - einschließlich des Pragmatismus selbst - einer permanenten Veränderung unterworfen ist. Der Pragmatismus ist deshalb grundsätzlich wissenschaftstheoretisch und methodologisch offen für plurale Wege sich der Komplexität unserer Welt zu nähern, solange diese undogmatisch bleiben. Alles, was sich bewährt, um nachvollziehbare Erkenntnisse über das ungeordnete und komplexe Chaos unseres Daseins in der Welt zu gewinnen, verdient für ihn Anerkennung (Dewey, 1996:LW4.176).

Auch wenn insofern nicht die eine pragmatische MenschUmwelt-Geographie geschrieben werden kann, regt der Pragmatismus doch zu spezifischen neuen Denkweisen, Forschungsperspektiven, Fragestellungen und Methodologien in Bezug auf Mensch-Umwelt-Beziehungen an. Den Drehund Angelpunkt eines pragmatisch-transaktionistischen Verständnisses der Beziehungen von Mensch und Umwelt bildet dabei, wie gezeigt, die Idee der Body-Minds, die als konstitutive Bedingung menschlichen Erfahrung-Machens und Prozessierens mit der Welt angesehen werden kann. Sie ist nicht nur geeignet Brücken zur ANT mit ihrem Konzept der dynamischen Hybriden (bspw. Jöns, 2003:119) zu schlagen, sondern weist auch Nähen zur Praxistheorie (bspw. Everts et al., 2011) in Bezug auf die konzeptionelle Rolle von Emotionen, Materialität und Wissen im Rahmen von Praktiken auf. Vor allem aber deckt sie sich weitgehend mit der phänomenologischen Idee des Leibes als eines fühlenden, beseelten Körpers (vgl. bspw. Merleau-Ponty, 1974). Der Leib bildet dabei - wie im Pragmatismus - die Bedingung der Welterfahrung des handelnden, wahrnehmenden und fühlenden Sub- jektes (Lindemann, 2011:591). Die Leiblichkeit des Menschen, um in der phänomenologischen Terminologie zu bleiben, ist es denn auch, die dafür verantwortlich ist, dass der Pragmatismus Mensch und Umwelt als holistische Gesamtheit im Sinne eines transaktiven ,organism-in-environmentas-a-whole" betrachtet. Dass wir Teil dieser Welt sind, erfahren wir letztlich über den Umstand des bewussten leiblichen Prozessierens mit ihr. Es ist dieser leibliche Charakter des menschlichen In-der-Welt-Seins, der die Absurdität der künstlichen dualistischen Trennung von Körper und Geist veranschaulicht und Menschen mit ihrer Verstandestätigkeit wieder materiell, sensual und emotional in der Welt verankert, der ihnen verdeutlich, dass sie Teil der Natur sind, denn „der Leib ist die Natur, die wir selbst sind“ (Böhme, 2011:554). Eine pragmatische Perspektive bietet sich deshalb als metatheoretischen Bezugspunkt auch für phänomenologische Ansätze in der Geographie an und könnte dazu bspw. anschließen an die Arbeiten von Hasse (bspw. 2003), der Plessners und Böhmes phänomenologische Leibphilosophie positioniert als Gegenmodell zu einem übersteigerten Sozialkonstruktivismus mit seinen (einseitig) „logozentristischen Erklärungsmodellen von Geschehnissen in der sozialen Wirklichkeit" (ebd.:27). Wie jüngst Korf (2012) deutlich gemacht hat immunisiert eine leibliche Perspektive aber auch gegen neuro-biologische Determinismen, die Handlungen allein durch neuronale Prozesse erklären zu können meinen und damit letztlich die Existenz des freien Willens und jeder Art von Intentionalität menschlicher Handlungen in Abrede stellen und so menschliches In-der-Welt-Agieren auf eine Affekt-Ebene reduzieren. Wie Korf (ebd.) demonstriert, wohnt auch der gegenwärtig stark diskutierten NRT von Nigel Thrift ein solcher anti-intentionaler Affekt-Begriff inne, der immer mehr auch in die deutschsprachige Debatte einzusickern scheint (bspw. Strüver, 2011). Eine solche neuro-deterministische Sicht ignoriert aber, sowohl aus leiblicher wie pragmatischer Perspektive, letztlich die holistische Verwobenheit und Gleichzeitigkeit des menschlichen Transagierens mit und in der Welt. Phänomenologie und Pragmatismus sind insofern mit ihrer Zurückweisung realistischer und konstruktivistischer Positionen durchaus aneinander anschlussfähig und argumentieren in die gleiche Richtung. Mit Hilfe des Pragmatismus ist es deshalb möglich einer phänomenologischen Perspektive einer „Geographie des Menschen" (Korf, 2012:159) auch eine nichtdualistische metatheoretische Basis jenseits von Idealismus oder Realismus anzubieten.

Nimmt man eine solche nichtdualistische und holistische Perspektive auf Mensch-Umwelt-Beziehungen ein, ergibt es logischerweise auch keinen Sinn, wie in Teilen der konstruktivistischen Humangeographie, Diskurse und Sprachspiele $\mathrm{zu}$ entwirklichen und zu hypostasieren, indem ihnen eine eigenständige Existenz unabhängig von den ihnen zugrunde liegenden Erfahrungsprozessen von Subjekten unterstellt wird. Um Missverständnissen vorzubeugen: Nichts würde Dewey ferner liegen als der Entleiblichung 
vieler poststrukturalistischer und konstruktivistischer Ansätze einen dumpfen Naturalismus entgegenzusetzen, der blind für symbolische Ordnungen und Logik ist. Dewey plädiert jedoch für eine Philosophie, die Logik nicht als etwas von Natur Getrenntes betrachtet, um sie als Apriori der Forschung zu postulieren, die in die vermeintlich ontologisch separierte Sphäre einer Welt der Ideen verweist. Vielmehr sieht er Logik als in Kontinuität mit der Gesamtheit der Kontexte stehend, mit denen Organismus und Umwelt in Transaktion stehen (Dewey and Bentley, 1949:LW.16.138). Die SpracheWelt-Beziehung ist demnach eine, die der Natur im Zuge des transaktiven Erfahrung-Machens inhärent ist und ihr nicht antipodisch gegenübergestellt werden kann. Für sprachpragmatische Ansätze, die wie Zierhofer (1997) und Schlottmann (2007) darum ringen zu einer Überwindung dualistischer Mensch-Umwelt-Konzeptionen beizutragen, könnte es daher fruchtbar sein, zu den semiotischen Konzepten des klassischen Pragmatismus zurückzukehren, die Sprache und Erfahrung zusammendenken und es so ermöglichen den „Sprachkäfig“ (Berwing, 2012) vieler poststrukturalistischer Ansätze zu verlassen.

Die leibliche Verankerung des Menschen in der Welt hat zur Konsequenz, dass Umwelt neu gedacht werden muss. Gleicht sie einem Medium oder Milieu, in dem und durch die Menschen leben, kann sie nichts mehr dem Menschen rein Externes sein, sondern bildet einen integralen und mit ihm transaktiv verwobenen Teil seiner Existenz. Mit einer solchen Abkehr von einer substanzialistischen hin zu einer prozessualen Bestimmung des Mensch-Umwelt-Verhältnisses ist dann aber das gängige Subjekt-Objekt-Denken unangemessen, in dem Umwelt als dem Menschen gegenüber gedacht wird. Den Menschen als Teil des prozessualen Ganzen zu verstehen, dezentriert ihn deshalb in der Welt. In einer solchen Denkweise erscheint es konsequent, sich von dem Begriff der Umwelt zu trennen und ihn durch den Begriff der Mitwelt zu ersetzen (bspw. Meyer-Abich, 1988). Die Mitwelt kann dann in pragmatischer Perspektive verstanden werden als die Gesamtheit der räumlich und zeitlich situierten Situationen, Ereignisse, Aktivitäten und Objekte, die mit einem Organismus transaktiv verbunden ist. Sie vereint in ihrer holistischen Anlage Physisch-Materielles mit Körperlichem, Emotionalem, Psychischem, Sozio-Politischem und Kulturellem und ist dasjenige, auf das sich alle Arten praktischer (inklusive kognitiver) Forschungsprozesse mit ihren physischen und physiologischen Implikationen ausrichten. Eine pragmatische Perspektive auf die Welt schließt daher alle Formen transaktionaler Beziehungen im Prozess individueller, leiblicher Erfahrungen mit ein. Neben den etablierten materie- oder sinnzentrierten Logiken sensibilisiert eine Veränderung der metatheoretischen Basis so für die Relevanz von Gefühlen und der sinnlichen Wahrnehmungen, verdeutlicht die metatheoretische Legitimation der wissenschaftlichen Beschäftigung mit diesen Gegenständen und bietet daher auch nicht-repräsentationalen Theorien ein metatheoretisches Zuhause. Das Transaktionskonzept ermög- licht es insofern auch, die Inkonsistenzen der NRT im Spannungsfeld zwischen ihren pragmatischen Wurzeln und ihres neuro-biologischen Affektkonzeptes aufzulösen, ohne dabei die Rolle von Affekten und Gefühlen für das menschliche Agieren in Abrede zu stellen.

Gibt man die Außenperspektive auf Umwelt auf und denkt die Welt als Mitwelt, lässt sich auch die moralische Sonderstellung des Menschen in der Welt nicht mehr halten, die ihn legitimiert seine Umwelt wie ein Objekt, wie pure Verfügungsmasse, zu behandeln (Mutschler, 2002:211). Auf der ethischen Ebene stellt sich daraus abgeleitet die Frage, ob der Mitwelt nicht ein Eigenwert im Ganzen und nicht nur ein Wert für den Menschen zugebilligt werden muss (Meyer-Abich, 1988:138). Eine Neuverortung des Menschen im Kosmos führt so auch zu der ethischen Frage des Umgangs mit der Mitwelt. Die Mitwelt als solche anzuerkennen bedeutet dabei nicht zwangsläufig Differenzen zwischen Menschlichem und Nichtmenschlichem egalisieren zu wollen (ebd.:136). Eine Ungleichbehandlung von Teilen der Mitwelt wird aber begründungsbedürftig, sie wird zu einer ethisch-normativen Frage (ebd.:137). Diese Frage ist natürlich hoch politisch, denn sie hat Konsequenzen dafür, wie wir mit Natur umgehen. Lässt man sich auf eine MenschMitwelt-Geographie ein, stellt sich deshalb letztlich die Frage, ob es unter solchen Vorzeichen Nicht-Politische Ökologien überhaupt geben kann. Im Gegensatz zu der normativen Enthaltsamkeit kritisch-rationalistischer und dekonstruktivistischer Positionen bietet sich eine pragmatische Perspektive deshalb gerade für eine normativ engagierte MenschMitwelt-Forschung an - bspw. auch, aber eben nicht nur, in der Nachhaltigkeitsdebatte.

Wenn Dinge nicht mehr substanzialistisch sondern transaktiv-prozessual als zeitlich und räumlich ausgedehnte Ereignisse in einem Prozess permanenten Wandels gedacht werden, erhält unsere Mitwelt inklusive dessen, was wir gewohnt sind Natur zu nennen, eine individuelle geschichtliche Dimension. Eine solche historisierende Perspektive führt logisch zu einer Art zeitlichen und räumlichen ,Vereinzelung der Wirklichkeit“, zu einer ,Zersplitterung des Weltblocks in für sich stehende Individualitäten, die sich nicht wiederholen“ (Hampe, 2011:291f) - und das gilt dann nicht nur für die Individualität der menschlichen Existenz, sondern für alle Ereignisse in der Mitwelt. Es ist denn auch diese Sicht auf Welt als eine Kette von Ereignissen, die der Pragmatismus ebenfalls mit der NRT teilt.

Auch die Perspektive von Welt als Ereignis birgt erhebliche wissenschaftstheoretische und methodologische Konsequenzen, muss man sich mit ihr doch von einer interaktionistischen Idee von Gleichgewichtssystemen und teleologischen Vorstellungen von Entwicklung zugunsten der Ideen kontinuierlicher und grundsätzlich unvorhersehbarer Veränderungen in einem komplexen Ganzen verabschieden. Insofern wäre zu fragen, ob sich die Transaktionstheorie nicht auch als meta-theoretische Grundlage für Kompexitätstheorien (Dikau, 2006) anbietet. In methodologischer Hinsicht 
ist die Geographie mit einer solchen prozessual-historischen Perspektive schließlich besonders gefordert, sich mit raumzeitlichen Besonderheiten und individuellen Konfigurationen von Ereignissen auseinander zu setzen, denn sie erlauben es uns schließlich überhaupt unterschiedliche Geographien unserer Mitwelt auszudifferenzieren. Sich nicht mit den Individualitäten der Welt zu beschäftigen, würde die Geographie direkt auf den Weg der abstrakten Wissenschaften, wie der Physik oder der Mathematik, führen. Wissenschaftstheoretisch gesprochen erfordert eine transaktive Perspektive deshalb eine Stärkung idiosynkratischer Ansätze in der geographischen Forschung, für deren Anwendung in der Geomorphologie jüngst bereits Kersting (2012) plädiert hat. Hierbei stellt sich der Disziplin allerdings wiederum die Frage, wie idiosynkratischen Perspektiven mehr Raum gegeben werden kann, ohne dass sie in alte Konzepte, wie die der Landschaftsgeographie, zurückzufallen droht.

Anders als man vermuten könnte schließt eine solche Aufmerksamkeitsverschiebung m.E. eine nomothetische Forschung jedoch keineswegs aus. Dies wäre im Sinne der undogmatischen Haltung des Pragmatismus auch kontraproduktiv. Gefordert wäre aber eine nomothetische Forschung, die von konkreten und in diesem Sinne besonderen und individuellen empirischen Gegenständen ausgeht und sich darüber bewusst ist, dass ihre Theorien und Modelle über Regularitäten und Zusammenhänge immer Abstraktionen und Vereinfachungen der chaotischen Wirklichkeit bleiben und die sich deshalb davor hütet ihre Theorien und Modelle zu hypostasieren, ihnen also eine eigenständige Existenz jenseits ihrer Entstehungskontexte zuzuschreiben.

In disziplintheoretischer Hinsicht führt eine transaktive Mitwelt-Perspektive schließlich dazu, dass die Unterscheidung von Physio- und Humangeographie ernsthaft hinterfragt werden muss, unterliegt dieser Perspektive doch die Vorstellung von zwei klar ontologisch zu unterscheidenden Gegenstandbereichen, die sich vor dem Hintergrund eines holistischen Mitweltkonzeptes aber nicht mehr aufrecht erhalten lässt. Dass humangeographische Fragen immer auch eine leibliche Dimension haben, mag dabei schnell einzusehen sein. Dass aber auch die Vorgänge, die wir üblicherweise als rein ,natürlich“ betrachten und die Gegenstand der Physiogeographie sind, so tief von der transaktiven Beziehung von Mensch und Mitwelt durchdrungen werden, dass es fraglich geworden ist, ob sich menschlich unbeeinflusste, rein „,natürliche“, Ökosysteme überhaupt noch finden lassen, mag sich nicht unmittelbar erschließen. Genau dies wird jedoch zunehmend im Rahmen der empirischen Debatte zur Idee eines Anthropozäns diskutiert (bspw. Crutzen, 2002; Braje and Erlandson, 2013; Ruddiman, 2003). Unabhängig davon, ob man den Begriff des Anthropozäns für sinnvoll erachtet, verdichten sich dabei offensichtlich die empirischen Hinweise darauf, wie sehr das globale Ökosystem bereits seit vielen tausend Jahren durch die transaktive Beziehung zwischen Mensch und Mitwelt geprägt wird.
Die Aufteilung der Disziplin in Frage zu stellen bedeutet natürlich nicht, dass man sich nicht weiterhin mit unterschiedlichen Fragen und Gegenständen beschäftigen könnte. Die Gliederung der Geographie in Teildisziplinen lässt sich dann aber nicht mehr aus der vermeintlichen ontologischen Unterschiedlichkeit unserer Erkenntnisobjekte, sondern nur noch analytisch begründen. Die ontologische Grundlage für den Graben in unserer Disziplin löst sich auf, wenn wir realistische und konstruktivistische zugunsten pragmatischer Perspektiven hinter uns lassen. Aus einer zweigeteilten Disziplin mit einer dritten Säule als Brücke wird eine holistisch verstandene Geographie der Mitwelt, die sich schlicht für unterschiedliche, eher sozial- oder naturwissenschaftliche Problemstellungen interessiert - aber nicht vergisst, dass diese in einen größeren Ereigniskontext eingebettet sind.

Die Konsequenzen einer pragmatisch-transaktionistischen Perspektive berühren daher eher die Denkweisen, Forschungsperspektiven, Fragestellungen, Methodologien und das Selbstverständnis der Geographie, als dass sie sich in ein spezifisches Theoriegebäude oder eine bestimmte Methodik übersetzen ließen. Sie weisen auf eine fruchtbare Einheit geographischer Forschung hin, ohne die Sinnhaftigkeit separater Bearbeitungsstrategien für zu untersuchende Teilprobleme mit ontologischen Argumenten zu begründen oder abzustreiten. Der Pragmatismus bietet sich deshalb als undogmatische, meta-theoretische Basis für eine nichtdualistische Mensch-Mitwelt-Forschung an, mit der es gelingen könnte die Gräben hinter sich zu lassen, in denen sich Realisten und Konstruktivisten verschanzt haben.

Acknowledgements. Teile dieses Textes wurden während des Passauer Geographentages 2013 und während der GeographieWerkstatt Österreich 2014 zur Diskussion gestellt. In diesem Kontext danke ich Martin Coy für die freundliche Einladung nach Innsbruck und den Zuhörern der beiden Vorträge für ihre kritischen Kommentare. Sie haben - ebenso wie die hilfreichen und konstruktiven Hinweise der Gutachter und Herausgeber - erheblich dazu beigetragen, meine Argumentation weiter zu schärfen.

Edited by: B. Korf

Reviewed by: three anonymous referees

\section{Literatur}

Austin, J. L.: How to Do Things with Words, Harvard University Press, Cambridge (MA), 1962.

Baker, V. R.: The Pragmatic Roots of American Quaternary Geology and Geomorphology, Geomorphology, 16, 197-215, 1996.

Barnes, T. J.: American Pragmatism: Towards a Geographical Introduction, Geoforum, 39, 1542-1554, 2008.

Becker, E. and Jahn, T.: Soziale Ökologie. Grundzüge einer Wissenschaft von den gesellschaftlichen Naturverhältnissen, Campus, Frankfurt/Main, 2006.

Berwing, S.: Jenseits des Sprachkäfigs: Potenziale der Peirce'schen Semiotik für eine Foucault'sche Kulturgeographie, Berichte zur Deutschen Landeskunde, 87, 67-81, 2012. 
Böhme, G.: Der Begriff des Leibes: Die Natur, die wir selbst sind, Dtsch. Z. Philos., 59, 553-563, 2011.

Braje, T. J. and Erlandson, J. M.: Human acceleration of animal and plant extinctions: A Late Pleistocene, Holocene, and Anthropocene Continuum, Anthropocene, doi:10.1016/j.ancene.2013.08.003, in press, 2013.

Bridge, G.: City Senses: On the Radical Possibilities of Pragmatism in Geography, Geoforum, 39, 1570-1584, 2008.

Cutchin, M. P.: John Dewey's Metaphysical Ground-Map and its Implications for Geographical Inquiry, Geoforum, 39, 15551569, 2008.

Crutzen, P. J.: Geology of Mankind, Nature, 415, p. 23, 2002.

Descola, P.: Jenseits von Natur und Kultur, Suhrkamp, Berlin, 2011.

Dewey, J.: Experience and Nature, Chicago, Open Court, 1925.

Dewey, J.: Logic: The Theory of Inquiry, New York, Holt, 1938.

Dewey, J.: Body and Mind (orginal 1927), in: The Collected Works of John Dewey, 1882-1953 (electronic edition) edited by: Boydston, J. A. and Hickmann, L. A., InteLex, Charlottesville, LW.3.25-40, 1996.

Dewey, J. and Bentley, A. F.: Knowing and the Known, Boston, Beacon, 1949.

Dewey, J.: The Collected Works of John Dewey, 1882-1953 (electronic edition), InteLex, Charlottesville, 1996.

Dikau, R.: Komplexe Systeme in der Geomorphologie, Mitt. Osterr. Geogr. G., 148, 125-150, 2006.

Everts, J., Lahr-Kurten, M., and Watson, M.: Practice matters! Geographical Inquiry and Theories of Practice, Erdkunde, 65, 323334, 2011.

Falter, R. and Hasse, J.: Geographie und das Mensch-NaturVerhältnis, Erdkunde, 56, 81-94, 2002.

Garrison, J.: An Introduction to Dewey's Theory of Functional "Trans-Action": An Alternative Paradigm for Activity Theory, Mind Culture and Activity, 8, 275-296, 2001.

Graefe, O., Korf, B., and Schlottmann, A.: Einführung in das Themenheft Natur und Gesellschaft - Neue Theorien in kritischer Sichtung, Geogr. Z., 89, 191-193, 2010.

Hampe, M.: Erkenntnis und Praxis. Zur Philosophie des Pragmatismus, Suhrkamp, Frankfurt am Main, 2006.

Hampe, M.: Tunguska oder das Ende der Natur, Hanser, München, 2011.

Hasse, J.: Die Frage nach den Menschenbildern - eine anthropologische Perspektive, in: Menschenbilder in der Humangeographie, edited by: Hasse, J. and Helbrecht, I., BIS, Oldenburg, 11-32, 2003.

Hepple, L. W.: Geography and the Pragmatic Tradition: The Threefold Engagement, Geoforum, 39, 1530-1541, 2008.

Hirsch, G.: Beziehungen zwischen Umweltforschung und disziplinärer Forschung, Gaia, 4, 302-314, 1995.

Hügli, A. and Lübcke, P. (Eds.): Philosophielexikon: Personen und Begriffe der abendländischen Philosophie von der Antike bis zur Gegenwart, Rowohlt, Reinbek bei Hamburg, 2005.

Hobson, K.: Environmental Responsibility and the Possibilities of Pragmatist-Orientated Research, Soc. Cult. Geogr., 7, 283-298, 2006.

Jöns, H.: Mensch-Umwelt-Beziehungen aus einer erweiterten Akteursnetzwerkperspektive, in: Humanökologie. Ansätze zur Überwindung der Natur-Kultur-Dichotomie, edited by: Meusburger, P. and Schwan, T., Erdkundliches Wissen, Franz Steiner, Wiesbaden, 103-137, 2003.
Kersting, P.: Geomorphologie, Pragmatismus und integrative Ansätze in der Geographie, Berichte zur Deutschen Landeskunde, 87, 49-65, 2012.

Korf, B.: Neuro-Kulturgeographie, Geogr. Z., 100, 146-163, 2012.

Latour, B.: The Force and the Reason of Experiment, in: Experimental Inquiries. Historical, Philosophical and Social Studies of Experimentation in Science, edited by: Le Grand, H. E., Kluwer, Dordrecht, 49-80, 1990.

Latour, B.: Der Berliner Schlüssel. Erkundungen eines Liebhabers der Wissenschaften, Berlin, 1996.

Latour, B.: Die Hoffnung der Pandora. Untersuchungen zur Wirklichkeit der Wissenschaft, Frankfurt/Main, Suhrkamp, 2002.

Latour, B.: Das Parlament der Dinge. Für eine politische Ökologie, Suhrkamp, Frankfurt/Main, Suhrkamp, 2010.

Latour, B.: Eine neue Soziologie für eine neue Gesellschaft. Einführung in die Akteur-Netzwerk-Theorie, Frankfurt/Main, Suhrkamp, 2012.

Lindemann, G.: Der menschliche Leib von der Mitwelt her gedacht, Dtsch. Z. Philos., 59, 591-604, 2011.

Menand, L.: The Metaphysical Club: A Study of Ideas in America, Farrar, Straus \& Giroux, New York, 2001.

Merleau-Ponty, M.: Phänomenologie der Wahrnehmung, deGruyter, Berlin, 1974.

Meyer-Abich, K. M.: Von der Umwelt Zur Mitwelt. Unterwegs zu einem neuen Selbstverständnis des Menschen im Ganzen der Natur, Scheidewege, 18, 128-148, 1988.

Mutschler, H. D.: Natur als Mitwelt. Holismus, Technisierung und Ausdrucksverhalten, in: Philosophie der natürlichen Mitwelt. Grundlagen-Probleme-Perspektiven, edited by: Ingensiep, $\mathrm{H}$. W., Eusterschulte, A., and Meyer-Abich, K. M., Königshausen \& Neumann, Würzburg, 211-226, 2002.

Meusburger, P. and Schwan, T. (Eds.): Humanökologie. Ansätze zur Überwindung der Natur-Kultur-Dichotomie, Franz Steiner, Wiesbaden, 2003.

Müller-Mahn, D. and Wardenga, U.: Möglichkeiten und Grenzen integrativer Forschungsansätze in Physischer Geographie und Humangeographie, Leipzig, Leibniz Institut für Länderkunde, 2005.

Proctor, J. D.: Geography, Paradox and Environmental Ethics, Prog. Hum. Geog., 22, 234-255, 1998a.

Proctor, J. D.: The Social Construction of Nature: Relativist Accusations, Pragmatist and Critical Realist Responses, Ann. Assoc. Am. Geogr., 88, 352-376, 1998 b.

Rorty, R.: Hoffnung statt Erkenntnis: Eine Einführung in die pragmatische Philosophie, Passagen, Wien, 1994.

Ruddiman, W. F.: The Anthropogenic Greenhouse Era Began Thousands of Years Ago, Clim. Change, 61, 261-293, 2003.

Schlottmann, A.: Wie aus Worten Orte werden. Gehalt und Grenzen sprechakttheoretischer Sozialgeographie, Geogr Z., 95, 523, 2007.

Schlottmann, A., Graefe, B., and Korf, B.: Things that Matter. A Dialogue on Interpretative and Material Semiotics in Geography, Geogr. Z., 89, 226-236, 2010.

Schütz, A. and Luckmann, T.: Strukturen der Lebenswelt, UVK Verlagsgesellschaft, Konstanz, 2003.

Steiner, C.: Materie oder Geist? Überlegungen zur Überwindung dualistischer Erkenntniskonzepte aus der Perspektive einer Pragmatischen Geographie, Berichte zur Deutschen Landeskunde, 83, 129-142, 2009. 
Strüver, A.: Der Konstruktivismus lernt laufen: "Doing more-thanrepresentational geography", Soc. Geogr., 6, 1-13, 2011, http://www.soc-geogr.net/6/1/2011/.

Thrift, N.: Non-Representational Theory: Space, Politics, Affect, Routledge, New York, 2008.

Wardenga, U. and Weichhart, P.: Sozialökologische Interaktionsmodelle und Systemtheorien - Ansätze einer theoretischen Begründung integrativer Projekte in der Geographie?, Mitt. Osterr. Geogr. G., 148, 9-31, 2006.

Weichhart, P.: Die transaktionistische Weltsicht - ein konzeptioneller Impuls für die Humanökologie? in: Perspektiven der Humanökologie, edited by: Kilchenmann, A. and Schwarz, C., Springer, Berlin, 227-238, 1991.

Weichhart, P.: How does the Person Fit into the Human Ecological Triangle? From Dualism to Duality: the Transactional Worldview, in: Human Ecology. Fragments of Anti-fragmentary Views of the World, edited by: Steiner, D. and Nauser, M., Routledge, London, 77-98, 1993.
Weichhart, P.: Auf der Suche nach der "dritten Säule”. Gibt es Wege von der Rhetorik zur Pragmatik? in: Möglichkeiten und Grenzen integrativer Forschungsansätze in Physischer Geographie und Humangeographie, edited by: Müller-Mahn, D. and Wardenga, U., ifl-forum, Institut für Länderkunde, Leipzig, 109-136, 2005.

Wescoat, J. L.: Common Themes in the Work of Gilbert White and John Dewey: A Pragmatic Appraisal, Ann Assoc. Am. Geogr., 82, 587-607, 1992.

Whatmore, S.: Hybrid Geographies: Natures, Culture, Spaces, Sage, London, 2002.

Wood, N. and Smith, S. J.: Pragmatism and Geography, Geoforum, 39, 1527-1529, 2008.

Zierhofer, W.: Grundlagen für eine Humangeographie des relationalen Weltbildes. Die sozialwissenschaftliche Bedeutung von Sprachpragmatik, Ökologie und Evolution, Erdkunde, 51, 81-99, 1997.

Zierhofer, W.: Geographie der Hybriden, Erdkunde, 53, 1-13, 1999. 